The effect of intraductal transanastomotic stent in reducing biliary complication after

\title{
duct-to-duct biliary reconstruction in living donor liver transplantation: Single center experience
}

\author{
Changho SEO, Ho Joong CHOI*, Sung Eun PARK, Joseph AHN, Tae Ho HONG, Young Kyoung YOU \\ Department of Surgery, Seoul St. Mary's Hospital, The Catholic University of Korea, Seoul, Korea
}

Introduction: Despite of the innovations in surgical and postoperative surgical treatment of donors and recipients, biliary complication is still considered to be a technical "Achilles' heel" of living donor liver transplantation (LDLT) due to the high incidence, requiring long-term interventional treatment, and potential risk for graft failure. The purpose of this study was to evaluate the effect of intraductal transanastomotic stent in reducing biliary complications after LDLT.

Methods: From August 2015 to February 2020, 201 adult LDLTs using right liver were enrolled. The intraductal transanastomotic stent was a silicone tube of various diameters considering the duct size. By dividing biliary complication into bile leakage and stricture, the risk factor and effect of stent were analyzed.

Results: In all patients with LDLT, biliary complications occurred in 54 (26.9\%) patients and anastomosis site leakage occurred in 9 (9.5\%) patients. Of the 201 patients, non-stent group was 101 (50.2\%) patients and stent group was 100 (49.8\%) patients. Anastomosis site leakage was higher in the non-stent group $(\mathrm{n}=15,14.9 \%)$ than in the stent group $(\mathrm{n}=4,4.0 \%, p=0.005)$. Biliary stricture was also higher in the non-stent group $(\mathrm{n}=30,29.7 \%)$ than in the stent group $(\mathrm{n}=17,17.0 \%, p=0.03)$. In multivariate analysis, hepatic artery thrombosis $(p<0.001)$ and intraductal stent $(p=0.01)$.

Conclusions: Intraductal transanastomotic stent can reduces biliary complications including anastomosis leakage and stricture. Further large-scale analyses of clinical data or randomized controlled trial are required to support this study. 